\title{
Enhancing the Long-Term Yield Competitiveness of a Semiconductor Manufacturing Factory Using a Multiobjective Fuzzy Nonlinear Programming Approach
}

\author{
Toly Chen and Yi-Chi Wang \\ Department of Industrial Engineering and Systems Management, Feng Chia University, No. 100 Wenhwa Road, Seatwen, \\ Taichung 407, Taiwan \\ Correspondence should be addressed to Toly Chen; tcchen@fcu.edu.tw
}

Received 9 March 2013; Revised 7 June 2013; Accepted 7 June 2013

Academic Editor: Jer-Guang Hsieh

Copyright (C) 2013 T. Chen and Y.-C. Wang. This is an open access article distributed under the Creative Commons Attribution License, which permits unrestricted use, distribution, and reproduction in any medium, provided the original work is properly cited.

This study proposes a multiobjective fuzzy nonlinear programming (MOFNP) approach to enhance the long-term yield competitiveness of a semiconductor manufacturing factory. By modeling the long-term competitiveness of every product in a semiconductor manufacturing plant with the fuzzy correlation coefficient (FCC) between time and instantaneous competitiveness, the proposed model considers the various viewpoints when interpreting the overall competitiveness of the semiconductor manufacturing plant in the long-term. All noninferior solutions of the MOFNP solutions are then derived using a systematic procedure. A real example is employed to illustrate the applicability of the proposed methodology.

\section{Introduction}

With the trend of globalization and the widespread applications of the Internet, the competition in many industries is becoming increasingly fierce. To survive in this environment, every firm must strive to continually improve its competence in one way or another. For example, some firms do not have their own factories, so that they can focus on activities that are more profitable, while others continue expanding their manufacturing capacity to further drive down costs. Some technologies that may be applied to improve the firm's chances of survival include the balanced scorecard, blue ocean strategy, green and lean technologies, supply chain management, and M. Porter's competitiveness diamond. Competitiveness enables a firm not only to survive, but also to increase its market share and profitability [1]. In his pioneering research, Porter defined the five forces influencing the competitiveness of a firm [2]. However, such forces are intangible, making it difficult to evaluate the competitiveness of a firm based on this concept. Nevertheless, they provide insightful directions for firms to enhance their competitiveness. Many studied that followed, including those of Armstrong [3], Jenkins et al. [4], Liao and Hu [5], Loch et al. [6], and Peng and Chien [7], were all devoted to the same purpose. They all concluded that continuous measurable improvement (in cycle time, product quality, on-time delivery, cost, and efficiency), statistical thinking, constraintfocus, people development/empowerment, knowledge transfer, differentiation, partnering, and collaboration through networking, as well as geographic reach are critical factors for the competitiveness of a firm. For a complete review please refer to Chen [8].

However, how to evaluate competitiveness is seldom being discussed in the literature. The traditional way to measure the competitiveness of a company is to interview stakeholders, such as past and present key management personnel, marketing and technological consultants, professional analysts, major capital equipment suppliers, and competitors $[1,9]$. This process is subjective and may lead to an imprecise assessment that can be unsuitable for quantitative analysis. Another frequently used approach is the hierarchical assessment approach, in which several aspects 
of competitiveness are being evaluated, and then a simple (weighted) averaging method is used to integrate the assessment results. However, this treatment is not based on practice and is questionable. Moreover, competitiveness is a subjective and uncertain concept, and the existing methods cannot maintain this level of flexibility. At the same time, quantitative measures such as market share $[1,10]$ and revenues $[1,10,11]$ are very sensitive to market conditions, beyond our control, and in need to be compared with those of the competitors or with the average in the entire industry. In addition, these measures are not the source but rather the outcomes of competitiveness. In short, competitiveness is a subjective and uncertain concept with multiple facets, such as quality, cost, responsiveness, on-time delivery, and others, and should be properly managed [11]. Recently, Chen [12] and Chen [8] both evaluated the yield (quality) competitiveness of a product from a semiconductor manufacturing factory. The concept was that if the quality of a product cannot reach a certain level before the required deadline, then the product is not considered to be competitive. In other words, the competitiveness of a product with respect to quality should be judged on a comparative basis. To model the uncertainty in the competitiveness concept, some researchers applied the fuzzy set theory. For example, Chen [8], Chen [13], and Chen and Wang [11] all forecasted the future yield of a product using a fuzzy learning process, which naturally resulted in a fuzzy-valued competitiveness that could be compared using linguistic terms such as "somewhat competitive" and "very uncompetitive" to reserve the flexibility in interpretation and implementation.

For the evaluation interval, Chen [12] defined the period for performing a short-term or midterm evaluation as one or two stages during the product life cycle. Afterwards, Chen [13] considered a period that was longer and included multiple check points to evaluate the long-term competitiveness of a semiconductor product. Chen [8] evaluated the longterm yield competitiveness of a semiconductor product by observing the competitiveness during the entire product life cycle which was represented with the fuzzy correlation coefficient (FCC) between the instantaneous competitiveness and time. Many methods have been proposed to calculate the fuzzy correlation coefficient [14-16]. Bustince and Burillo [14] extended the evaluation of the FCC between two interval-valued intuitionistic fuzzy sets. Hong and Hwang [15] investigated the same issue in probabilistic spaces. Most existing approaches in this field are based on the concept of informational energy, while Chen's approach [8] is based on $\alpha$-cut operations. Also, in previous studies, the FCC between two fuzzy variables was expressed with a crisp value, while in [8] the FCC between a fuzzy variable (the instantaneous competitiveness) and a crisp variable (time) was represented with a fuzzy value. Chen constructed a fuzzy nonlinear programming (FNP) model to improve the longterm yield competitiveness through capacity control that switched capacity among products.

Chen [13] and Chen and Wang [11] extended this concept and considered many products simultaneously in an attempt to evaluate the competitiveness of the entire factory. This is reasonable since a manufacturer is undoubtedly defined by its products [6]. They also showed that with a proper factory capacity control mechanism, the competitiveness of the entire factory can be efficiently enhanced.

In this study, we extended Chen's approach [8] to evaluate and enhance the long-term yield competitiveness of a semiconductor manufacturing factory. Today's competition in the semiconductor manufacturing industry has reached an unprecedented level of intensity. Typical products of this industry include dynamic random access memory (DRAM), flash, and application-specific integrated circuits. In order to maintain a sustainable development under these difficult circumstances, semiconductor manufacturers have come up with various ways to improve their competitiveness, such as alliances, becoming fabless, outsourcing, and developing next-generation technologies. Dr. Helms, CEO of International SEMATECH, once remarked, "In our industry it used to be that the big companies ate the small ones. Today, the fast ones run over the slow." [17]. Leachman [9] benchmarked ten semiconductor manufacturing facilities to identify the factors that influence competitive semiconductor manufacturing (CSM). The findings were classified into four categories: yield related, throughput related, productivity related, and cycle time related. Compared with previous studies, our proposed methodology has the following innovative characteristics.

(1) In past studies, the competitiveness of a factory was equal to the average value of its products that might have different ranges, while in the present study the FCC is used to express the long-term competitiveness which usually falls within $[-1,1]$ and facilitates a reasonable aggregation.

(2) Lately, the product life cycles of products have become shorter, and thus we utilized a new approximation formula that is more accurate than Chen and Wang's approach [11] for calculating the instantaneous competitiveness.

(3) All of the past studies considered only a singleobjective function when optimizing the effects of capacity control on competitiveness. There are in fact different issues that must be taken into consideration in real life, including maximizing the number of competitive products, the average competitiveness, or the weighted competitiveness, all of which result in different objective functions. For this reason, a multiobjective fuzzy nonlinear programming (MOFNP) model was constructed in this study to guide the capacity control actions.

The reasonability of the methodology comes from the following aspects.

(1) Yield is the most important index to the competitiveness of a factory in this industry.

(2) Yield is a learning phenomenon that can be observed and predicted under long-term observation and analysis.

(3) Multiple analyses of a problem from diverse perspectives raise the possibility that no relevant aspects of 
the problem will be ignored. Multiobjective optimization approaches may fit this requirement.

The remainder of this paper is organized as follows. Section 2 details the procedure for evaluating the long-term competitiveness of a semiconductor product using the FCC between the instantaneous competitiveness and time. An MOFNP model is then constructed in Section 3 to guide the capacity control actions to switch capacity among products. In Section 4, in order to facilitate solution finding, an equivalent NP model is established for the proposed MOFNP model that can be solved with any existing optimization software. To prove the usefulness of the proposed methodology, a real case from a DRAM manufacturing factory was investigated in Section 5. Finally, in Section 6, with a view towards the future, we provide a summary of our findings.

\section{Evaluating the Long-Term Yield Competitiveness of a Product}

Before evaluating the long-term yield competitiveness, we have to fit the fuzzy yield learning model:

$$
\tilde{Y}_{t}=\tilde{Y}_{0} e^{-(\tilde{b} / t)+r(t)},
$$

where $\widetilde{Y}_{t}$ is the instantaneous yield at time period $t, \widetilde{b}$ is the learning constant, and $\widetilde{Y}_{0}$ denotes the asymptotic/final yield.

For evaluating the long-term yield competitiveness of a product, multiple check points, denoted as $m_{(k)} ; k=1 \sim$ $K$, must be set up. Each check point is associated with a competitive region indicated as $\mathrm{CR}_{(k)}$, with $k=1 \sim K$ :

$$
\mathrm{CR}_{(k)}: \tilde{Y}_{t} \geq Y_{(k)}^{*} \quad \text { when } t \geq m_{(k)}, k=1 \sim K,
$$

where $Y_{(k)}^{*}$ is the yield target at check point $m_{(k)}$. The instantaneous competitiveness of the product at check point $k$ can then be assessed as

$$
\widetilde{\mathcal{c}}_{(k)}=\int_{m_{(k)}}^{m_{(k)}+\Delta t} \tilde{Y}_{t} d t-\int_{m_{(k)}}^{m_{(k)}+\Delta t} Y_{(k)}^{*} d t, \quad k=1 \sim K,
$$

where $\Delta t$ is a small positive value $(\leq 1)$ representing the time allowance for achieving $Y_{(k)}^{*}$. If $\Delta t=1$; then the calculation result is between $\left[-Y_{(k)}^{*}, 1-Y_{(k)}^{*}\right]$. A negative value of $\widetilde{c}_{(k)}$ implies the product is not competitive at that check point.

To evaluate the long-term competitiveness of a product, the instantaneous competitiveness trend can be observed, for which the FCC between the instantaneous competitiveness and time is derived as follows:

$$
\begin{gathered}
\widetilde{C}=\frac{\sum_{\text {all } k} m_{(k)} \widetilde{c}_{(k)}(-) K \bar{m} \overline{\widetilde{c}}}{\sqrt{\sum_{\text {all } k} m_{(k)}^{2}-K \bar{m}^{2}} \sqrt{\sum_{\text {all } k} \widetilde{c}_{(k)}^{2}(-) K \overline{\widetilde{c}}^{2}}} \\
\overline{\widetilde{c}}=\sum_{k=1}^{K} \frac{\widetilde{c}_{k}}{K} \\
\bar{m}=\sum_{k=1}^{K} \frac{m_{k}}{K},
\end{gathered}
$$

where $\widetilde{C}$ ranges from -1 to 1 . If $\widetilde{C}$ is negative; then the product is not long-term competitive. $\widetilde{C}$ can also be classified into the following categories, as in the classical correlation analysis:

"Very Uncompetitive" if $\widetilde{C}<-0.7$

"Somewhat Uncompetitive" if $-0.7 \leq \widetilde{C}<0$

"Moderate" if $\widetilde{C}$ is around 0

"Somewhat Competitive" if $0 \leq \widetilde{C}<0.7$

"Very Competitive" if $\widetilde{C} \geq-0.7$.

Equation (4) is not easy to calculate, and for this reason we applied $\alpha$-cut operations as follows

$$
\begin{aligned}
& A_{\alpha}=\left\{x \mid x \in R, \mu_{\widetilde{A}}(x) \geq \alpha\right\} \equiv \text { the } \alpha \text {-cut of } \widetilde{A}=\left[A_{1}^{\alpha}, A_{2}^{\alpha}\right] \\
& B_{\alpha}=\left\{x \mid x \in R, \mu_{\widetilde{B}}(x) \geq \alpha\right\} \equiv \text { the } \alpha \text {-cut of } \widetilde{B}=\left[B_{1}^{\alpha}, B_{2}^{\alpha}\right] .
\end{aligned}
$$

Then,

$$
\begin{aligned}
A_{\alpha}(+) B_{\alpha} & =\left[A_{1}^{\alpha}, A_{2}^{\alpha}\right](+)\left[B_{1}^{\alpha}, B_{2}^{\alpha}\right] \\
& =\left[A_{1}^{\alpha}+B_{1}^{\alpha}, A_{2}^{\alpha}+B_{2}^{\alpha}\right] \\
A_{\alpha}(-) B_{\alpha} & =\left[A_{1}^{\alpha}, A_{2}^{\alpha}\right](-)\left[B_{1}^{\alpha}, B_{2}^{\alpha}\right] \\
& =\left[A_{1}^{\alpha}-B_{2}^{\alpha}, A_{2}^{\alpha}-B_{1}^{\alpha}\right] \\
A_{\alpha}(\times) B_{\alpha} & =\left[A_{1}^{\alpha}, A_{2}^{\alpha}\right](\times)\left[B_{1}^{\alpha}, B_{2}^{\alpha}\right] \\
& =\left[A_{1}^{\alpha} \cdot B_{1}^{\alpha}, A_{2}^{\alpha} \cdot B_{2}^{\alpha}\right] ; \quad A_{1}^{\alpha} \geq 0 ; B_{1}^{\alpha} \geq 0 \\
A_{\alpha}(/) B_{\alpha} & =\left[A_{1}^{\alpha}, A_{2}^{\alpha}\right](/)\left[B_{1}^{\alpha}, B_{2}^{\alpha}\right] \\
& =\left[A_{1}^{\alpha} / B_{2}^{\alpha}, A_{2}^{\alpha} / B_{1}^{\alpha}\right] ; \quad A_{1}^{\alpha} \geq 0 ; B_{1}^{\alpha}>0,
\end{aligned}
$$

where $(+),(-),(\times)$, and $(/)$ denote fuzzy addition, subtraction, multiplication, and division, respectively.

The relationship between the instantaneous competitiveness of a product and its learning constant is an exponential function. A learning constant with a large value results in a low competitiveness [11]. In addition, the learning constant of a product is inversely proportional to the capacity allocated to the product. If we increase this capacity, say from $q$ to $q^{\prime}$, then the learning constant changes to $\widetilde{b}\left(q / q^{\prime}\right)$, and the instantaneous competitiveness of that product at check point $m_{(k)}$ can be elevated to

$$
\begin{aligned}
\tilde{c}_{(k)}^{\prime} & =\tilde{Y}_{0} \int_{m_{(k)}}^{m_{(k)}+\Delta t} e^{-\left(\widetilde{b}\left(q / q^{\prime}\right) / t\right)} d t-Y_{(k)}^{*} \Delta t \\
& =\tilde{Y}_{0} \int_{m_{(k)}}^{m_{(k)}+\Delta t} e^{-\left(\tilde{b}^{\prime} / t\right)} d t-Y_{(k)}^{*} \Delta t,
\end{aligned}
$$


where $\tilde{b}^{\prime}$ is the new learning constant after capacity reallocation and $\tilde{b}^{\prime}=\tilde{b} \cdot q / q^{\prime}$. As a result, the long-term competitiveness of the product changes to

$$
\widetilde{C}^{\prime}=\frac{\sum_{\text {all } k} m_{(k)} \vec{c}_{(k)}^{\prime}(-) K \bar{m} \overline{\bar{c}}^{\prime}}{\sqrt{\sum_{\text {all } k} m_{(k)}^{2}-K \bar{m}^{2}} \sqrt{\sum_{\text {all } k} \widetilde{c}_{(k)}^{\prime 2}(-) K \overline{\tilde{c}}^{\prime 2}}} .
$$

However, the increase in the instantaneous competitiveness does not usually elevate the long-term competitiveness, because this trend is not easy to change.

\section{Enhancing the Long-Term Yield Competitiveness of the Entire Factory: An MOFNP Model}

A semiconductor manufacturing factory usually has many products. If all of them are yield competitive, then the whole factory is undoubtedly yield competitive [18]. Conversely, if some products are competitive while others are not, then the competitiveness of the entire factory is questionable, especially if less competitive products are the majority of the capacity. To prevent that, we can cease the production of less competitive products or allocate more capacity to enhance the competitiveness of such products, with the overall goal being the optimization of the competitiveness of the entire factory.

Assume that currently there are $L$ products being manufactured in the factory. The final yield and the learning constant of product $l$ are denoted as $\tilde{Y}_{0}(l)$ and $\tilde{b}(l)$, respectively; $l=1 \sim L$. The competitive regions for product $l$ to achieve are denoted with $\left(m_{(k)}(l), Y_{(k)}^{*}(l)\right) ; k=1 \sim K$. The long-term competitiveness of product $l, \widetilde{C}(l)$, can then be evaluated according to (3) as

$$
\widetilde{C}(l)=\frac{\sum_{\text {all } k} m_{(k)}(l) \widetilde{c}_{(k)}(l)(-) K \bar{m}(l) \overline{\widetilde{c}}(l)}{\sqrt{\sum_{\text {all } k} m_{(k)}^{2}(l)-K \bar{m}^{2}(l)} \sqrt{\sum_{\text {all } k} \widetilde{c}_{(k)}^{2}(l)(-) K \overline{\tilde{c}}^{2}(l)}} .
$$

Assuming that the capacity originally allocated for making product $l$ is $q(l)$, and the capacity of these products is $Q$, then

$$
\sum_{l=1}^{L} q(l)=Q
$$

After reallocating the capacity, the capacity for product $l$ becomes $q^{\prime}(l)$, and its evaluated competitiveness changes to $\widetilde{C}^{\prime}(l)$ according to $(10)$,

$$
\widetilde{C}^{\prime}(l)=\frac{\sum_{\text {all } k} m_{(k)}(l) \tilde{c}_{(k)}^{\prime}(l)(-) K \bar{m}(l) \overline{\tilde{c}}^{\prime}(l)}{\sqrt{\sum_{\text {all } k} m_{(k)}^{2}(l)-K \bar{m}^{2}(l)} \sqrt{\sum_{\text {all } k} \widetilde{c}_{(k)}^{\prime}(l)(-) K \overline{\tilde{c}}^{\prime}(l)^{2}}},
$$

where

$$
\begin{gathered}
\overrightarrow{\mathcal{c}}_{(k)}^{\prime}(l)=\tilde{Y}_{0}(l) \int_{m_{(k)}(l)}^{m_{(k)}(l)+\Delta t} e^{-\left(\widetilde{b}(l) q(l) / q^{\prime}(l) t\right)} d t-Y_{(k)}^{*}(l) \Delta t \\
=\widetilde{Y}_{0}(l) \int_{m_{(k)}(l)}^{m_{(k)}(l)+\Delta t} e^{-\left(\widetilde{b}^{\prime}(l) / t\right)} d t-Y_{(k)}^{*}(l) \Delta t \\
\tilde{b}^{\prime}(l)=\frac{\widetilde{b}(l) \cdot q(l)}{q^{\prime}(l)} .
\end{gathered}
$$

Also,

$$
\sum_{l=1}^{L} q^{\prime}(l)=Q
$$

The competitiveness of the entire factory can be measured in several ways, for example, the average competitiveness, the minimal competitiveness, the weighted competitiveness, the number of competitive products, the number of highly competitive products, and so forth. In this study, the focus is on the average competitiveness and the number of competitive products. In addition, a capacity reallocation plan that causes little disturbance is used. Taking these factors into consideration, the proposed MOFNP model is formulated to maximize the competitiveness of the semiconductor manufacturing factory through capacity re-allocation:

$$
\begin{array}{ll}
\text { Maximize } & Z_{1}=\sum_{l=1}^{L} \frac{\widetilde{C}^{\prime}(l)}{L} \\
\text { Maximize } & Z_{2}=\sum_{l=1}^{L} P(l)
\end{array}
$$

$$
\begin{gathered}
\text { s.t. } \quad P(l)=\left\{\begin{array}{ll}
1 & \text { if } \widetilde{C}^{\prime}(l) \geq 0 \\
0 & \text { otherwise }
\end{array} ; \quad l=1 \sim L\right. \\
\widetilde{C}^{\prime}(l)=\frac{\sum_{\text {all } k} m_{(k)}(l) \widetilde{c}_{(k)}^{\prime}(l)(-) K \bar{m}(l) \overline{\bar{c}}^{\prime}(l)}{\sqrt{\sum_{\text {all } k} m_{(k)}^{2}(l)-K \bar{m}^{2}(l)} \sqrt{\sum_{\text {all } k} \widetilde{c}_{(k)}^{\prime}(l)(-) K \overline{\tilde{c}}^{\prime}(l)^{2}}} ; \\
\quad l=1 \sim L
\end{gathered}
$$

$$
\begin{gathered}
\widetilde{c}_{(k)}^{\prime}(l)=\tilde{Y}_{0}(l) \int_{m_{(k)}(l)}^{m_{(k)}(l)+\Delta t} e^{-\left(\widetilde{b}^{\prime}(l) / t\right)} d t-Y_{(k)}^{*}(l) \Delta t \\
\sum_{l=1}^{L} q^{\prime}(l)=Q \\
\Delta q(l)=q^{\prime}(l)-q(l) \\
\sum_{l=1}^{L}|\Delta q(l)| \leq \omega \\
q^{\prime}(l) \in Z^{+} ; \quad l=1 \sim L,
\end{gathered}
$$


TABLE 1: The replacement mechanism.

\begin{tabular}{lcc}
\hline If & Then & So \\
\hline$\sum_{\alpha=0}^{1} \frac{C_{L}^{\prime}(l)(\alpha)+C_{R}^{\prime}(l)(\alpha)}{21}<0$ & $P(l)-0.5 \leq 0$ & $P(l)=0$ \\
$\sum_{\alpha=0}^{1} \frac{C_{L}^{\prime}(l)(\alpha)+C_{R}^{\prime}(l)(\alpha)}{21}=0$ & $\begin{array}{c}\text { No restriction } \\
\text { on } P(l)-0.5\end{array}$ & $P(l)=0$ or 1 \\
$\sum_{\alpha=0}^{1} \frac{C_{L}^{\prime}(l)(\alpha)+C_{R}^{\prime}(l)(\alpha)}{21}>0$ & $P(l)-0.5 \geq 0$ & $P(l)=1$ \\
\hline
\end{tabular}

where $\omega \in Z^{+}$is an upper bound for the scale of capacity re-allocation. If $P(l)=1$, then product $l$ is long-term competitive. All variables are given in triangular fuzzy numbers (TFNs). The proposed FNP model is intractable and may need to be converted into an equivalent NP problem. In the literature, Loukil et al. [19] mentioned the following five ways used to deal with multiobjective optimization problems.

(1) Simultaneous (or Pareto) approach: their combination should be formed in such a way that ensures that the performances along different dimensions are Pareto optimal. For minimization problems with $K$ objective functions, if the value of objective function $f_{k}$ can only be decreased by increasing the value of some other objective function $f_{j} ; k \neq j ; j, k \in$ $\{1, \ldots, M\}$, then all feasible solutions that fulfill this property are called Pareto optimal solutions.

(2) Utility (or compromise) approach: to simplify the finding of the best solution, the linear or nonlinear combination of the objectives is going to be optimized instead, the so-called compromise solution. For example, the new objective function can be a weighted sum of the multiple objectives of the original multi-objective problem. The different solutions for the noninferior set are obtained by changing the weights.

(3) Goal programming (or satisfying) approach: some objectives are formulated as constraints, for which the satisfaction levels are specified. For example, for a maximization problem, all but one of the objectives are converted into constraints of the form

$$
Z_{k} \geq G_{k}
$$

The noninferior solutions can be obtained by solving many single-objective optimization problems with different values of vector $G$. The Kuhn-Tucker conditions of optimality for both (2) and (3) have been proved.

(4) Hierarchical approach: objectives are sequentially achieved but not at the same time.
(5) Interactive approach: a number of steps are required, and the decision maker expresses his/her preferences to the solution proposed at each step to evolve it to the most acceptable one.

\section{Converting into an Equivalent NP Problem}

The theoretical part for the proposed methodology is the goal programming approach described in the previous section. In this study, we determine all the Pareto optimal solutions in the following manner.

Step 1. $G_{2}=0$.

Step 2. Find out the optimal solution with a single objective function $Z_{1}$ and an additional constraint $Z_{2} \geq G_{2}$.

Step 3. $G_{2}=G_{2}+1$. If $G_{2}>L$, stop; otherwise, go to Step 2 .

This treatment is also beneficial for the flexibility required when implementing the capacity re-allocation plan.

Considering the complex shape of $\widetilde{C}^{\prime}(l)$, the $\alpha$-cut operations are applied to convert it into an equivalent crisp value. We assume that the left and right margins of the $\alpha$ cuts of $\widetilde{C}^{\prime}(l)$ are denoted as $C_{L}^{\prime}(l)(\alpha)$ and $C_{R}^{\prime}(l)(\alpha) ; \alpha=0,0.1, \ldots, 1$. Then, (18) can be substituted with

$$
P(l)= \begin{cases}1 & \text { if } \sum_{\alpha=0}^{1} \frac{C_{L}^{\prime}(l)(\alpha)+C_{R}^{\prime}(l)(\alpha)}{21} \geq 0 ; \quad l=1 \sim L . \\ 0 & \text { otherwise }\end{cases}
$$

In addition, (26) can be replaced with the following constraints:

$$
\begin{array}{r}
(P(l)-0.5) \cdot \sum_{\alpha=0}^{1} \frac{C_{L}^{\prime}(l)(\alpha)+C_{R}^{\prime}(l)(\alpha)}{21} \geq 0 \\
P(l) \in\{0,1\} .
\end{array}
$$

Table 1 is used to demonstrate such a replacement. In (26), the denominator of the left-hand side is 21 because eleven values of $\alpha:\{0,0.1, \ldots, 1\}$ are considered. When $\alpha$ is $1, C_{L}^{\prime}(\alpha)=$ $C_{R}^{\prime}(l)(\alpha)$, so only $2 * 11-1=21$ different values are calculated. Subsequently, we separate $\widetilde{c}_{(k)}^{\prime}(l)$ into two sets:

$$
\begin{aligned}
& Q_{1}(l)=\left\{\widetilde{c}_{(k)}^{\prime}(l) \text { 's at their left margins of the } \alpha \text { cuts }\right\} \\
& Q_{2}(l)=\left\{\widetilde{c}_{(k)}^{\prime}(l) \text { 's at their right margins of the } \alpha \text { cuts }\right\} .
\end{aligned}
$$

After applying the $\alpha$-cut operations to (19), $C_{L}^{\prime}(l)(\alpha)$ and $C_{R}^{\prime}(l)(\alpha)$ can be obtained as follows:

$$
C_{L}^{\prime}(l)(\alpha)=\min _{\text {all } \mathbb{Q}_{1}(l), Q_{2}(l)}\left(\frac{\sum_{k \in Q_{1}(l)} m_{(k)} c^{\prime}{ }_{(k)}(l)(\alpha)+\sum_{k \in Q_{2}(l)} m_{(k)} c^{\prime}{ }_{(k)}(l)(\alpha)-\bar{m}\left(\sum_{k \in Q_{1}(l)} c^{\prime}{ }_{(k)}(l)(\alpha)+\sum_{k \in Q_{2}(l)} c^{\prime}(k)(l)(\alpha)\right)}{\sqrt{\sum_{\text {all } k} m_{(k)}^{2}-K \bar{m}^{2}} \sqrt{\sum_{k \in Q_{1}} c^{\prime}{ }_{(k)}(l)(\alpha)^{2}+\sum_{k \in Q_{2}} c^{\prime}{ }_{(k)}(l)(\alpha)^{2}-\left(\sum_{k \in Q_{1}} c^{\prime}{ }_{(k)}(l)(\alpha)+\sum_{k \in Q_{2}} c^{\prime}{ }_{(k)}(l)(\alpha)\right)^{2} / K}}\right),
$$




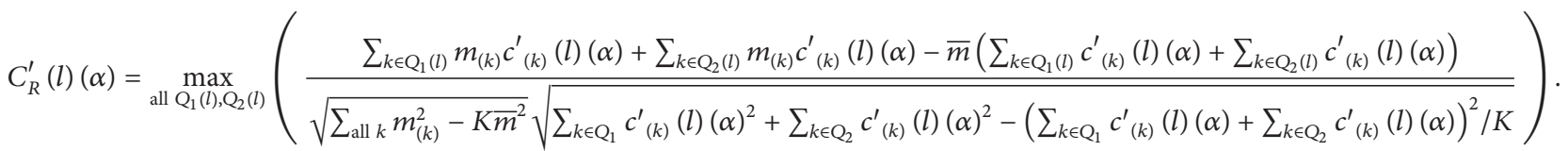

These equations can be easily rewritten without the $\max / \mathrm{min}$ function. For example, (28) becomes

$$
C_{L}^{\prime}(l)(\alpha) \leq \frac{\sum_{k \in \mathrm{Q}_{1}(l)} m_{(k)} c^{\prime}{ }_{(k)}(l)(\alpha)+\sum_{k \in \mathrm{Q}_{2}(l)} m_{(k)} c^{\prime}{ }_{(k)}(l)(\alpha)-\bar{m}\left(\sum_{k \in \mathrm{Q}_{1}(l)} c_{(k)}^{\prime}(l)(\alpha)+\sum_{k \in \mathrm{Q}_{2}(l)} c_{(k)}^{\prime}(l)(\alpha)\right)}{\sqrt{\sum_{\text {all } k} m_{(k)}^{2}-K \bar{m}^{2}} \sqrt{\sum_{k \in \mathrm{Q}_{1}} c^{\prime}{ }_{(k)}(l)(\alpha)^{2}+\sum_{k \in \mathrm{Q}_{2}} c_{(k)}^{\prime}(l)(\alpha)^{2}-\left(\sum_{k \in \mathrm{Q}_{1}} c^{\prime}{ }_{(k)}(l)(\alpha)+\sum_{k \in \mathrm{Q}_{2}} c_{(k)}^{\prime}(l)(\alpha)\right)^{2} / K}}
$$

for all $Q_{1}(l)$ 's and $Q_{2}(l)$ 's, and

$$
\prod_{\text {all } \mathrm{Q}_{1}(l), \mathrm{Q}_{2}(l)}\left(C_{L}^{\prime}(l)(\alpha)-\frac{\sum_{k \in \mathrm{Q}_{1}(l)} m_{(k)} c^{\prime}{ }_{(k)}(l)(\alpha)+\sum_{k \in \mathrm{Q}_{2}(l)} m_{(k)} c^{\prime}{ }_{(k)}(l)(\alpha)-\bar{m}\left(\sum_{k \in \mathrm{Q}_{1}(l)} c^{\prime}{ }_{(k)}(l)(\alpha)+\sum_{k \in \mathrm{Q}_{2}(l)} c^{\prime}{ }_{(k)}(l)(\alpha)\right)}{\sqrt{\sum_{\text {all } k} m_{(k)}^{2}-K \bar{m}^{2}} \sqrt{\sum_{k \in \mathrm{Q}_{1}} c^{\prime}{ }_{(k)}(l)(\alpha)^{2}+\sum_{k \in \mathrm{Q}_{2}} c^{\prime}{ }_{(k)}(l)(\alpha)^{2}-\left(\sum_{k \in \mathrm{Q}_{1}} c^{\prime}{ }_{(k)}(l)(\alpha)+\sum_{k \in \mathrm{Q}_{2}} c^{\prime}{ }_{(k)}(l)(\alpha)\right)^{2} / K}}\right)=0 .
$$

Subsequently, in order to convert (20), Chen and Wang [11] derived the Taylor series approximation of $e^{-1 / t}$ within interval $[10,120]$. However, most new products do not last that long, and therefore in this study we derived it within interval $[10,60]$. To be precise

$$
\begin{array}{r}
e^{-1 / t} \cong-3.7 \cdot 10^{-8} t^{4}+7 \cdot 10^{-6} t^{3}-0.00047 t^{2} \\
+0.0141 t+0.8065, \quad 10 \leq t \leq 60 .
\end{array}
$$

The mean absolute percentage error (MAPE) with such an approximation is only $0.2 \%$. Now, let $\widetilde{b}^{\prime}(l)=\widetilde{b}(l) \cdot q(l) / q^{\prime}(l)$ and replace $t$ with $s \widetilde{b}^{\prime}(l)$. Then, after differentiation,

$$
d t=\widetilde{b}^{\prime}(l) d s
$$

We then substitute (32) and (33) into equation (20), which then becomes

$$
\begin{aligned}
& \widetilde{c}_{(k)}^{\prime}(l) \\
& =\tilde{Y}_{0}(l) \int_{m_{(k)}(l) / \tilde{b}^{\prime}(l)}^{\left(m_{(k)}(l)+\Delta t\right) / \tilde{b}^{\prime}(l)} \tilde{b}^{\prime}(l) e^{-1 / s} d s-Y_{(k)}^{*}(l) \Delta t \\
& =\tilde{Y}_{0}(l) \tilde{b}^{\prime}(l) \int_{m_{(k)}(l) / \tilde{b}^{\prime}(l)}^{\left(m_{(k)}(l)+\Delta t\right) / \tilde{b}^{\prime}(l)}\left(-3.7 \cdot 10^{-8} s^{4}+7 \cdot 10^{-6} s^{3}\right. \\
& -0.00047 s^{2}+0.0141 s \\
& +0.8065) d s-Y_{(k)}^{*}(l) \Delta t \\
& =\tilde{Y}_{0}(l) \tilde{b}^{\prime}(l)\left(-0.74 \cdot 10^{-8} s^{5}+1.75 \cdot 10^{-6} s^{4}-0.00016 s^{3}\right. \\
& \left.+0.0071 s^{2}+0.8065 s\right)\left.\right|_{m_{(k)}(l) / b^{\prime}(l)} ^{\left(m_{(k)}(l)+\Delta t\right) / \tilde{b}^{\prime}(l)}
\end{aligned}
$$$$
-Y_{(k)}^{*}(l) \Delta t
$$

$$
\begin{aligned}
= & \tilde{Y}_{0}(l) \tilde{b}^{\prime}(l) \\
\times(- & 0.74 \cdot 10^{-8}\left(\frac{m_{(k)}(l)+\Delta t}{\widetilde{b}^{\prime}(l)}\right)^{5} \\
& +1.75 \cdot 10^{-6}\left(\frac{m_{(k)}(l)+\Delta t}{\widetilde{b}^{\prime}(l)}\right)^{4} \\
& -0.00016\left(\frac{m_{(k)}(l)+\Delta t}{\widetilde{b}^{\prime}(l)}\right)^{3} \\
& +0.0071\left(\frac{m_{(k)}(l)+\Delta t}{\widetilde{b}^{\prime}(l)}\right)^{2} \\
& +0.8065\left(\frac{m_{(k)}(l)+\Delta t}{\widetilde{b}^{\prime}(l)}\right)^{+}+0.74 \cdot 10^{-8}\left(\frac{m_{(k)}(l)}{\widetilde{b}^{\prime}(l)}\right)^{5} \\
& -1.75 \cdot 10^{-6}\left(\frac{m_{(k)}(l)}{\widetilde{b}^{\prime}(l)}\right)^{4}+0.00016\left(\frac{m_{(k)}(l)}{\widetilde{b}^{\prime}(l)}\right)^{3} \\
-Y_{(k)}^{*} & (l) \Delta t . \\
& \left.-0.0071\left(\frac{m_{(k)}(l)}{\widetilde{b}^{\prime}(l)}\right)^{2}-0.8065\left(\frac{m_{(k)}(l)}{\widetilde{b}^{\prime}(l)}\right)\right)^{5}
\end{aligned}
$$

Equation (34) can be substituted with the following equations:

$$
\begin{aligned}
& c_{(k)}^{\prime L}(l)(\alpha) \\
& \quad=Y_{0}^{L}(l)(\alpha)\left(-0.74 \cdot 10^{-8} \frac{\left(m_{(k)}(l)+\Delta t\right)^{5}}{b^{\prime R}(l)(\alpha)^{4}}\right.
\end{aligned}
$$




$$
\begin{aligned}
& +1.75 \cdot 10^{-6} \frac{\left(m_{(k)}(l)+\Delta t\right)^{4}}{b^{\prime R}(l)(\alpha)^{3}} \\
& -0.00016 \frac{\left(m_{(k)}(l)+\Delta t\right)^{3}}{b^{\prime R}(l)(\alpha)^{2}} \\
& +0.0071 \frac{\left(m_{(k)}(l)+\Delta t\right)^{2}}{b^{\prime R}(l)(\alpha)} \\
& +0.8065\left(m_{(k)}(l)+\Delta t\right) \\
& +0.74 \cdot 10^{-8} \frac{m_{(k)}(l)^{5}}{b^{\prime R}(l)(\alpha)^{4}} \\
& -1.75 \cdot 10^{-6} \frac{m_{(k)}(l)^{4}}{b^{\prime R}(l)(\alpha)^{3}} \\
& +0.00016 \frac{m_{(k)}(l)^{3}}{b^{\prime R}(l)(\alpha)^{2}}-0.0071 \frac{m_{(k)}(l)^{2}}{b^{\prime R}(l)(\alpha)} \\
& \left.-0.8065 m_{(k)}(l)\right)-Y_{(k)}^{*}(l) \Delta t
\end{aligned}
$$

$c_{(k)}^{\prime R}(l)(\alpha)$

$$
\begin{aligned}
& =Y_{0}^{R}(l)(\alpha)\left(-0.74 \cdot 10^{-8} \frac{\left(m_{(k)}(l)+\Delta t\right)^{5}}{b^{L}(l)(\alpha)^{4}}\right. \\
& +1.75 \cdot 10^{-6} \frac{\left(m_{(k)}(l)+\Delta t\right)^{4}}{b^{L}(l)(\alpha)^{3}} \\
& -0.00016 \frac{\left(m_{(k)}(l)+\Delta t\right)^{3}}{b^{L}(l)(\alpha)^{2}} \\
& +0.0071 \frac{\left(m_{(k)}(l)+\Delta t\right)^{2}}{b^{L}(l)(\alpha)} \\
& +0.8065\left(m_{(k)}(l)+\Delta t\right) \\
& +0.74 \cdot 10^{-8} \frac{m_{(k)}(l)^{5}}{b^{L}(l)(\alpha)^{4}} \\
& -1.75 \cdot 10^{-6} \frac{m_{(k)}(l)^{4}}{b^{\prime L}(l)(\alpha)^{3}} \\
& +0.00016 \frac{m_{(k)}(l)^{3}}{b^{L}(l)(\alpha)^{2}}-0.0071 \frac{m_{(k)}(l)^{2}}{b^{L}(l)(\alpha)} \\
& \left.-0.8065 m_{(k)}(l)\right)-Y_{(k)}^{*}(l) \Delta t,
\end{aligned}
$$

where $\left\{Y_{0}^{L}(l)(\alpha), Y_{0}^{R}(l)(\alpha)\right\}$ and $\left\{b^{\prime L}(l)(\alpha), b^{\prime R}(l)(\alpha)\right\}$ denote the $\{$ left margin of the $\alpha$-cut, right margin of the $\alpha$-cut $\}$ of $\tilde{Y}_{0}(l)$ and $\tilde{b}^{\prime}(l)$, respectively. Note that $\widetilde{c}_{(k)}^{\prime}(l)$ is a monotonic decreasing function of $\widetilde{b}^{\prime}(l)$ that is obtained as follows:

$$
\begin{aligned}
& b^{\prime L}(l)(\alpha)=b^{L}(l)(\alpha) \cdot \frac{q(l)}{q^{\prime}(l)} \\
& b^{\prime R}(l)(\alpha)=b^{R}(l)(\alpha) \cdot \frac{q(l)}{q^{\prime}(l)} .
\end{aligned}
$$

Finally, the equivalent NP problem can be solved using any existing optimization software.

\section{A Practical Example}

To illustrate the applicability of the proposed methodology, a real case from a 12-inch wafer fabrication factory was used. Currently, wafers are fabricated using the $30 \mathrm{~nm}$ technology in the wafer fabrication factory. Although the target factory has yet to evaluate its competitiveness in a quantitative way, it does consider competitiveness an important issue, for which an agreeable solution is bound to turn up eventually. Currently, there are more than 10 types of memory chips, including dynamic random access memory (DRAM) and flash chips, being fabricated in this semiconductor manufacturing factory. Among them, the data of three major product types, indicated as $A, B$, and $C$, were collected. In Table 2 , the yields for the three products for the first 8 periods were summarized. The factory capacity is about 24000 wafers per month, which are distributed among its products in the following way:

Product $A$ : about 12000 wafers per month; that is, $q(1)=8000$

Product $B$ : about 7200 wafers per month; that is, $q(2)=3500$

Product C: about 7200 wafers per month; that is, $q(3)=9500$

Other products: about 3000 wafers per month

$Q=8000+3500+9500=21000$. After applying Chen and Wang's approach, the fuzzy yield learning models of these products were obtained as

$$
\begin{gathered}
\widetilde{Y}_{t}(A)=(0.83,1,1) e^{-(0.734,0.734,0.734) / t+r(t)} \\
\widetilde{Y}_{t}(B)=(0.85,0.85,1) e^{-(0.182,0.182,0.182) / t+r(t)} \\
\widetilde{Y}_{t}(C)=(0.815,0.815,0.815) e^{-(0.064,0.064,1.240) / t+r(t)} .
\end{gathered}
$$

Please note that all parameters are given in TFNs. The minimal satisfaction level was set to be 0.3 . It is well known that a higher level of satisfaction leads to a fuzzy yield with a wider range (see Figure 1). According to Figure 1, the yield converges to only $80 \%$ that may be a bit lower than expected. The yield model may need to be refitted when there are more data. In addition, some improvement may be made on the yield learning through certain of quality 


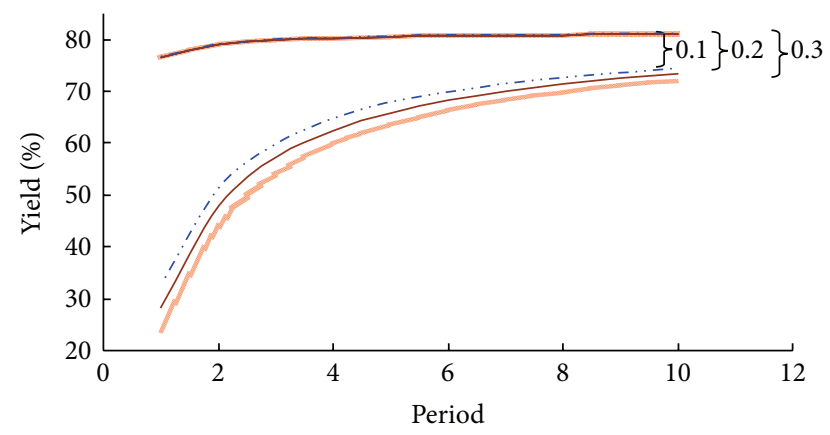

Figure 1: The effects of the minimal satisfaction level (product $C$ ).

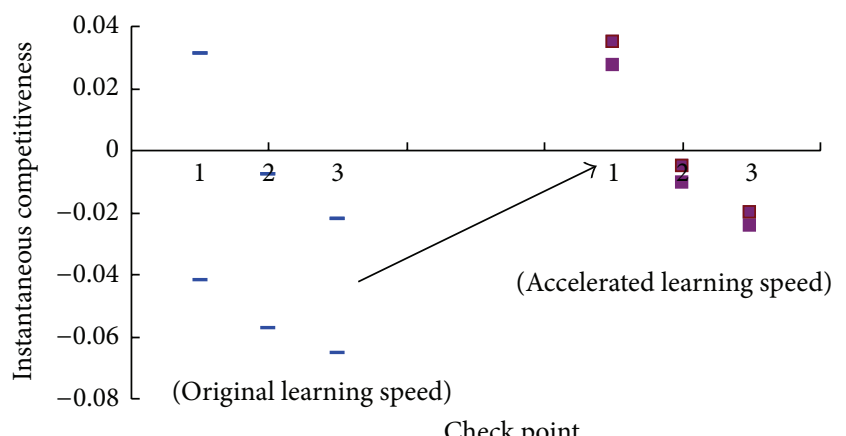

FIGURE 2: Increasing the instantaneous competitiveness by accelerating yield learning (product $C$ ).

activities. The competitive regions for evaluating the longterm competitiveness of these products were decided by their corresponding product engineers, as summarized in Table 3. For example, the average yield for product $B$ has to be greater than $83.7 \%$ to take the edge for competition at period 14. In practical applications, it is not necessary to check the competitiveness of all products simultaneously. Then, the instantaneous competitiveness for each product was evaluated at every check point based on (3). The results were summarized in Table 4. Accelerating the learning speed (and reducing the learning constant) was beneficial to the instantaneous competitiveness, as shown in Figure 2.

The original FCCs of these products were derived according to (4) (see Figure 3). Since they were of different shapes they could not be approximated with simple TFNs. We also examined the effects of accelerating the yield learning process on the long-term competitiveness expressed with the FCC. The result is shown in Figure 4, indicating that the FCC did not grow with accelerated yield learning, which is reasonable since increasing the points on a line does not always increase the slope of the line. In Chen [13], the longterm yield competitiveness was equal to the average of the instantaneous ones. The results of [13] (the average method) and the proposed methodology (the FCC method) were compared in Figure 5. It is evident that they do not pair up with each other. Theoretically, the uncertainty involved in a long-term period should be greater than that within a short period. Therefore, the FCC approach proposed in this study

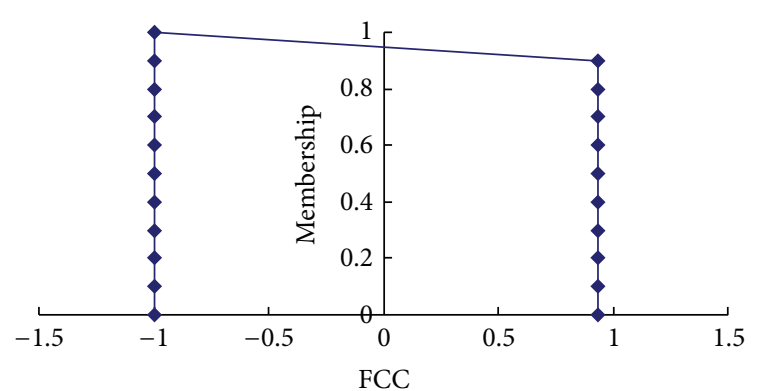

(a) Product $A$

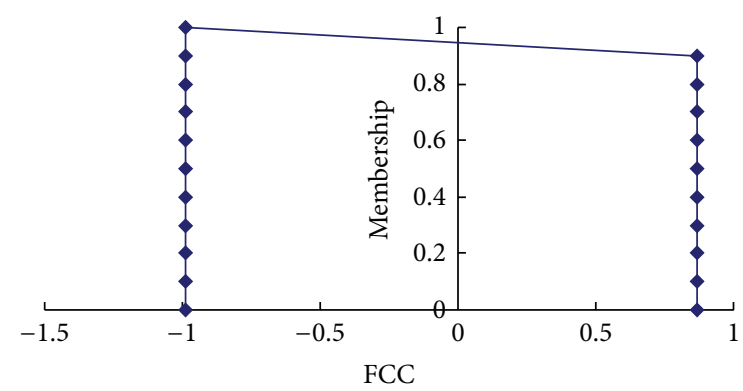

(b) Product $B$

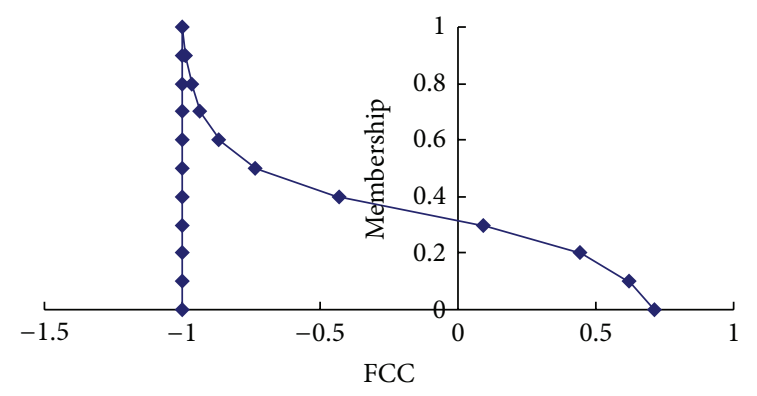

(c) Product $C$

FIGURE 3: The FCCs of the three products.

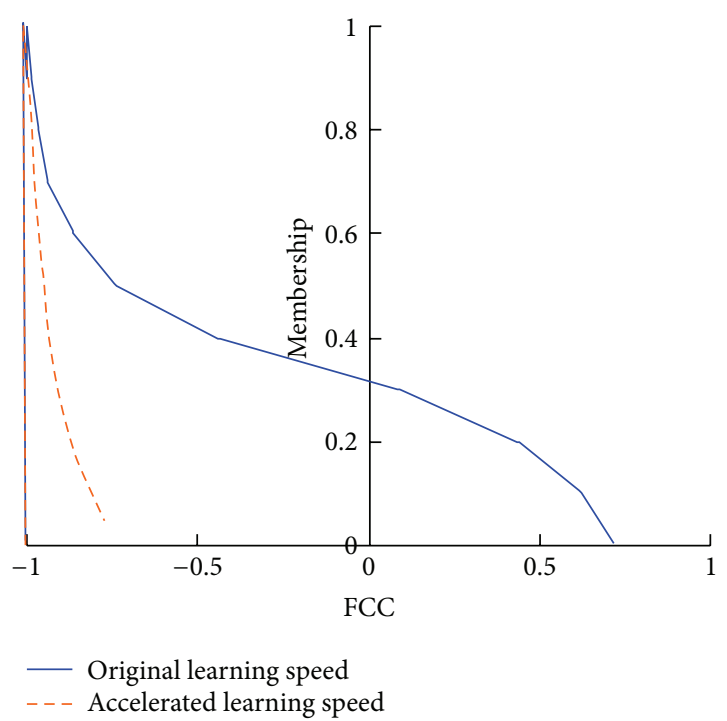

Figure 4: The effects of accelerating yield learning on the FCC. 
TABLE 2: A practical example.

\begin{tabular}{lccc}
\hline Period & Product $A$ & Product $B$ & Product $C$ \\
\hline 1 & - & $79.6 \%$ & $76.5 \%$ \\
2 & - & $81.2 \%$ & $71.1 \%$ \\
3 & - & $84.2 \%$ & $61.7 \%$ \\
4 & $73.3 \%$ & $84.7 \%$ & $73.3 \%$ \\
5 & $80.5 \%$ & $82.0 \%$ & $79.2 \%$ \\
6 & $84.0 \%$ & - & $78.4 \%$ \\
7 & $79.3 \%$ & - & $80.8 \%$ \\
8 & $81.7 \%$ & - & $80.7 \%$ \\
\hline
\end{tabular}

TABLE 3: The competitive regions.

\begin{tabular}{lccc}
\hline Product & $\begin{array}{c}\text { Competitive } \\
\text { region number 1 }\end{array}$ & $\begin{array}{c}\text { Competitive } \\
\text { region number 2 }\end{array}$ & $\begin{array}{c}\text { Competitive } \\
\text { region number 3 }\end{array}$ \\
\hline$A$ & $(12,86 \%)$ & $(15,87.4 \%)$ & $(21,90 \%)$ \\
$B$ & $(14,83.7 \%)$ & $(17,85 \%)$ & $(21,88 \%)$ \\
$C$ & $(12,78 \%)$ & $(18,82 \%)$ & $(21,83.5 \%)$ \\
\hline
\end{tabular}

TABLE 4: The instantaneous competitiveness.

\begin{tabular}{lccc}
\hline Product & $\begin{array}{c}\text { Check point } \\
\text { number } 1\end{array}$ & $\begin{array}{c}\text { Check point } \\
\text { number 2 }\end{array}$ & $\begin{array}{c}\text { Check point } \\
\text { number 3 }\end{array}$ \\
\hline A & $(-0.077,0.083$, & $(-0.082,0.08$, & $(-0.098,0.066$, \\
& $0.083)$ & $0.08)$ & $0.066)$ \\
$B$ & $(0.002,0.002$, & $(-0.009,-0.009$, & $(-0.037,-0.037$, \\
& $0.151)$ & $0.14)$ & $0.112)$ \\
$C$ & $(-0.042,0.031$, & $(-0.058,-0.008$, & $(-0.066,-0.022$, \\
& $0.031)$ & $-0.008)$ & $-0.022)$ \\
\hline
\end{tabular}

provides a more reasonable evaluation than that of Chen's approach.

The convergence of the algorithm was described as follows. The original FNP model had two objective functions, 21 variables and 24 constraints. The decision variable $\left(q^{\prime}\right)$ was a positive integer, and therefore there were finite solutions. The upper bound on the capacity that could be transited $(\omega)$ was 20000 wafers per month. After conversion, the equivalent NP problem had two objective functions, 129 variables and 141 constraints. The NP problem was solved with LINGO on a PC with AMD Athlon 64 Processor $3000+1.8 \mathrm{GHz}$ and $1 \mathrm{G}$ RAM within a few minutes. To find out all possible Pareto optimal solutions, four goals for $Z_{2}$ were established with a new constraint and the problem could then be optimized for a single objective function $\left(Z_{1}\right)$ using the branch-and-bound algorithm. The results are summarized in Table 5. There were feasible solutions only when $Z_{2}$ was set to 0,1 , or 2 , and the optimal solutions were the same when $Z_{2}$ was 0 and 1 . As a result, only two Pareto optimal solutions were found.

Although it seems to be difficult to make all products competitive at the same time, the experimental results indicate that improving the overall competitiveness measured in terms of either the number of competitive products or the average competitiveness is possible. For example, after capacity reallocation, the number of competitive products changed

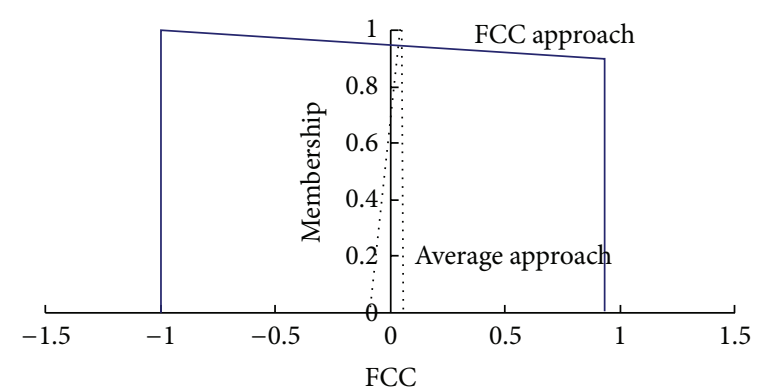

FIGURE 5: Two approaches for evaluating the long-term competitiveness.

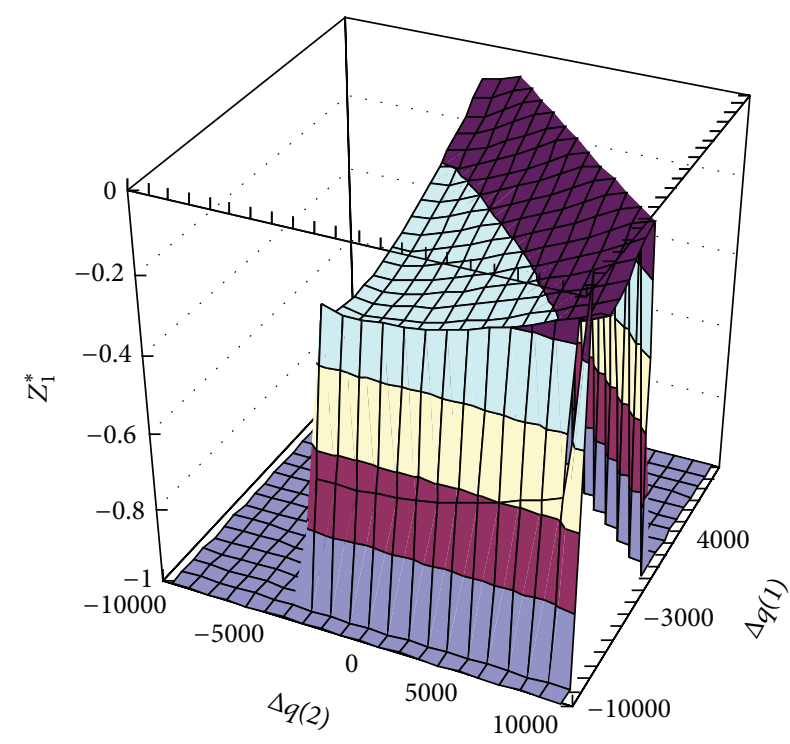

Figure 6: The effects of the additional capacity on $Z_{1}^{*}$.

TABLE 5: The optimization results.

\begin{tabular}{lcccccc}
\hline Round & $Z_{2}$ goal & $Z_{1}^{*}$ & $Z_{2}^{*}$ & $\Delta q_{1}^{*}$ & $\Delta q_{2}^{*}$ & $\Delta q_{3}^{*}$ \\
\hline 1 & 0 & 0.122 & 1 & -7843 & 10000 & -2157 \\
2 & 1 & 0.122 & 1 & -7843 & 10000 & -2157 \\
3 & 2 & 0.005 & 2 & 4626 & 4694 & -9320 \\
4 & 3 & NA & NA & NA & NA & NA \\
\hline
\end{tabular}

from 1 to 2 . At the same time, the average competitiveness could be increased by $145 \%$ (from -0.258 to 0.117 ).

The additional capacity allocated to each product is the most critical parameter. Therefore, some parameter analyses were conducted to assess the effect on the optimal solution. There were three parameters that summed up to a constant. Thus, we built up a 3D response surface chart with two independent variables to exploit their effect at the same time. From Figure 6, it is obvious that $Z_{1}^{*}$ grows with the increase in either parameter. The response surface in Figure 7 reveals that removing about 3000 wafers per month from product $B$ and distributing it among the other products is the most efficient way of improving $Z_{2}^{*}$. 
TABLE 6: The superiority of the proposed methodology over the existing methods.

\begin{tabular}{|c|c|c|c|c|c|}
\hline Method & $\begin{array}{c}\text { Number of objectives } \\
\text { considered }\end{array}$ & $\begin{array}{c}\text { Time interval that can } \\
\text { be evaluated }\end{array}$ & $\begin{array}{l}\text { Systematic and quantitative } \\
\text { competitiveness evaluation }\end{array}$ & $\begin{array}{l}\text { Considering the trend } \\
\text { in the competitiveness }\end{array}$ & $\begin{array}{c}\text { Considering the } \\
\text { uncertainty }\end{array}$ \\
\hline Leachman [9] & 4 & short term long term & No & No & No \\
\hline $\begin{array}{l}\text { Chen and } \\
\text { Wang [11] }\end{array}$ & 1 & short term $\sim$ mid term & Yes & No & Yes \\
\hline Chen [13] & 1 & short term long term & Yes & No & Yes \\
\hline $\begin{array}{l}\text { The proposed } \\
\text { methodology }\end{array}$ & 2 & short term long term & Yes & Yes & Yes \\
\hline
\end{tabular}

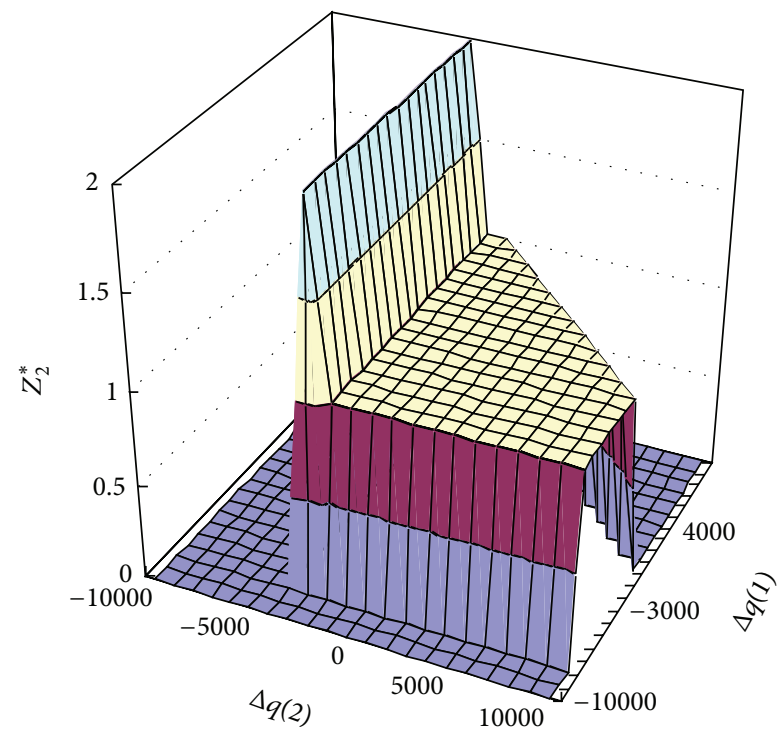

FIGURE 7: The effects of the additional capacity on $Z_{2}^{*}$.

In summary, when the factory capacity was allocated originally, the overall long-term competitiveness did not exist but could be enhanced after capacity reallocation. Of the two Pareto optimal solutions, the second solution caused less disturbance to the factory operations by retransmitting less capacity and therefore the production control staff considered it to be more practical.

\section{Conclusions and Directions for Future Research}

An MOFNP approach was proposed in this study for enhancing the long-term yield competitiveness of a semiconductor manufacturing factory. In the proposed methodology, the long-term competitiveness of every product in a semiconductor manufacturing factory was evaluated with the trend in the instantaneous competitiveness of the product, which was represented by the FCC between time and the instantaneous competitiveness. Therefore, capacity reallocation is considered to be helpful for enhancing long-term competitiveness. There are various viewpoints for interpreting the overall competitiveness of a semiconductor manufacturing plant when considering the performance of all its products. After incorporating all viewpoints, an MOFNP model was constructed to optimize the effects of capacity re-allocation on the competitiveness of the semiconductor manufacturing plant. To demonstrate the practicability of the proposed methodology, a detailed example using the practical data from a real semiconductor manufacturing plant was adopted. The findings from the experimental results were as follows.

(1) The semiconductor manufacturing factory was not competitive if its capacity was allocated in the original way. After capacity re-allocation, it was possible to elevate the average competitiveness by $145 \%$ and to make two main products more competitive.

(2) In total, three noninferior solutions were found, which reserved the flexibility for interpreting the competitiveness of the semiconductor manufacturing factory and allowed for flexibility when implementing the capacity reallocation plan.

(3) Fuzzy-valued competitiveness does indeed reflect the uncertainty in yield learning and in the concept itself.

The comparison of the proposed methodology with the existing methods is summarized in Table 6. However, this study might be criticized for the fact that the competitiveness was evaluated using a complicated optimization process. However, the work presented in this study can be extended to other facets of competitiveness.

\section{References}

[1] S. T. Walsh, R. L. Boylan, C. McDermott, and A. Paulson, "The semiconductor silicon industry roadmap: epochs driven by the dynamics between disruptive technologies and core competencies," Technological Forecasting and Social Change, vol. 72, no. 2, pp. 213-236, 2005.

[2] M. E. Porter, "How competitive forces shape strategy," Harvard Business Review, 1979.

[3] E. Armstrong, "Principles for competitive semiconductor manufacturing," in Proceedings of the IEEE/SEMI International Semiconductor Manufacturing Science Symposium (ISMSS '89), pp. 67-72, May 1989.

[4] T. Jenkins, F. Phail, and S. Sackman, "Semiconductor competitiveness in the 1990s," in Proceedings of the Society of Automotive Engineers Conference, pp. 249-255, October 1990.

[5] S.-H. Liao and T.-C. Hu, "Knowledge transfer and competitive advantage on environmental uncertainty: an empirical study of the Taiwan semiconductor industry," Technovation, vol. 27, no. 6-7, pp. 402-411, 2007. 
[6] C. H. Loch, S. Chick, and A. Huchzermeier, Management Quality and Competitiveness, Springer, Berlin, Germany, 2008.

[7] C.-Y. Peng and C.-F. Chien, "Data value development to enhance competitive advantage: a retrospective study of EDA systems for semiconductor fabrication," International Journal of Services, Technology and Management, vol. 4, no. 4-6, pp. 365383, 2003.

[8] T. Chen, "A FNP approach for establishing the optimal and efficient capacity re-allocation plans for enhancing the longterm competitiveness of a semiconductor product," International Journal of Fuzzy Systems, vol. 12, no. 2, pp. 158-169, 2010.

[9] R. C. Leachman, Competitive Semiconductor Manufacturing: Final Report on Findings From Benchmarking Eight-Inch, Sub$350 \mathrm{Nm}$ Wafer Fabrication Lines, Berkley, New York, NY, USA, 2002.

[10] S. Deffree, "Semiconductor winners and losers split by competitiveness," 2007, http://www.electronicsweekly.com/.

[11] T. Chen and Y.-C. Wang, "A fuzzy set approach for evaluating and enhancing the mid-term competitiveness of a semiconductor factory," Fuzzy Sets and Systems, vol. 160, no. 5, pp. 569-585, 2009.

[12] T. Chen, "Evaluating the mid-term competitiveness of a product in a semiconductor fabrication factory with a systematic procedure," Computers and Industrial Engineering, vol. 53, no. 3, pp. 499-513, 2007.

[13] T. Chen, "A FNP approach for evaluating and enhancing the long-term competitiveness of a semiconductor fabrication factory through yield learning modeling," International Journal of Advanced Manufacturing Technology, vol. 40, no. 9-10, pp. 993-1003, 2009.

[14] H. Bustince and P. Burillo, "Correlation of interval-valued intuitionistic fuzzy sets," Fuzzy Sets and Systems, vol. 74, no. 2, pp. 237-244, 1995.

[15] D. H. Hong and S. Y. Hwang, "Correlation of intuitionistic fuzzy sets in probability spaces," Fuzzy Sets and Systems, vol. 75, no. 1, pp. 77-81, 1995.

[16] G. W. Wei, H. J. Wang, and R. Lin, "Application of correlation coefficient to interval-valued intuitionistic fuzzy multiple attribute decision-making with incomplete weight information," in Knowledge and Information Systems, Springer, London, UK, 2009.

[17] R. Helms, "Chips: the fastest downturn in history has its optimist," Business Week, 2001.

[18] J. A. Cunningham, "Use and evaluation of yield models in integrated circuit manufacturing," IEEE Transactions on Semiconductor Manufacturing, vol. 3, no. 2, pp. 60-71, 1990.

[19] T. Loukil, J. Teghem, and D. Tuyttens, "Solving multi-objective production scheduling problems using metaheuristics," European Journal of Operational Research, vol. 161, no. 1, pp. 42-61, 2005. 


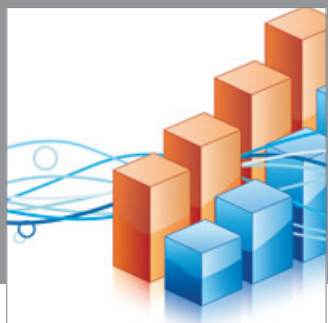

Advances in

Operations Research

mansans

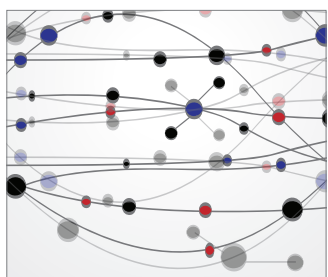

The Scientific World Journal
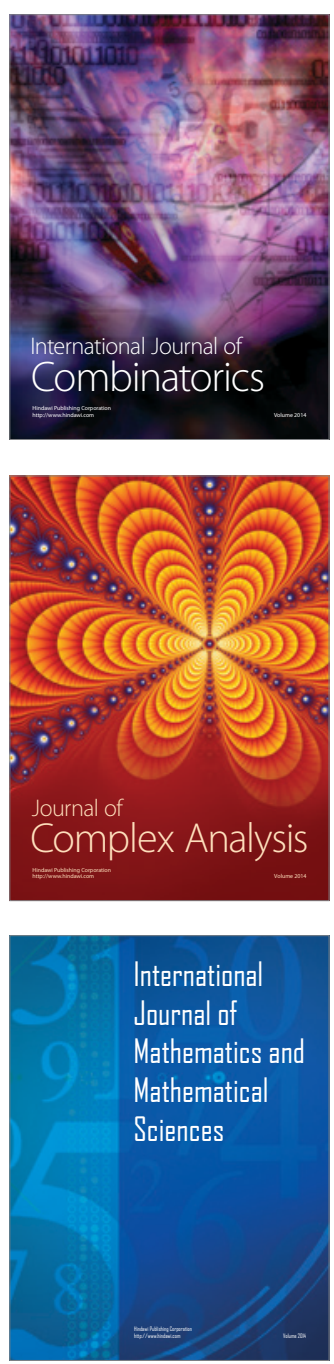
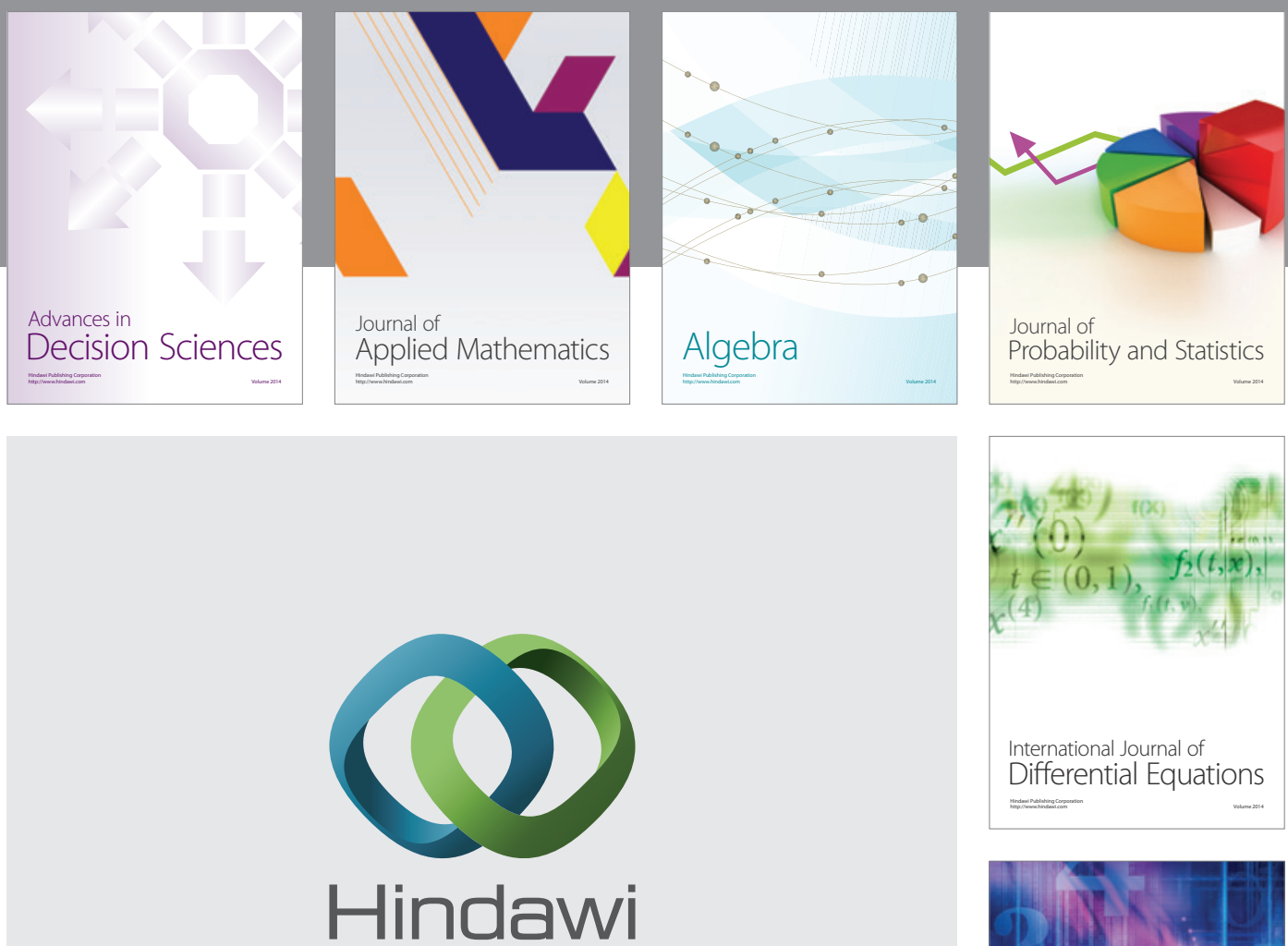

Submit your manuscripts at http://www.hindawi.com
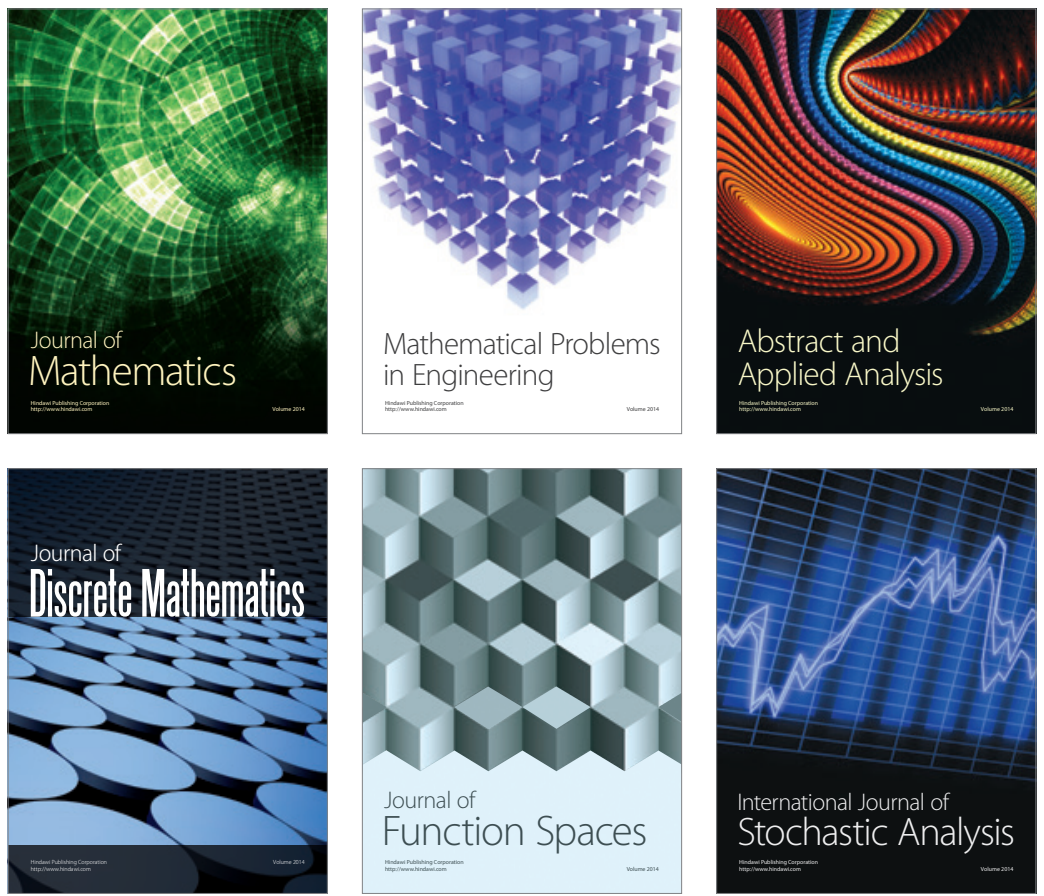

Journal of

Function Spaces

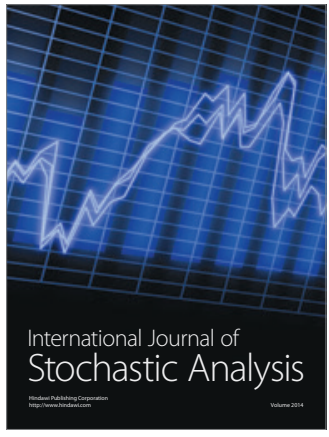

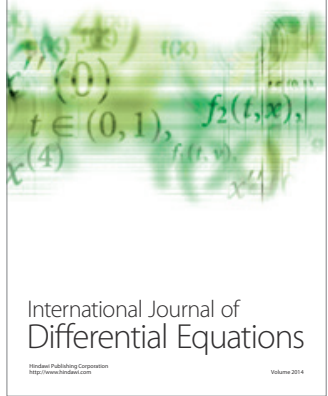
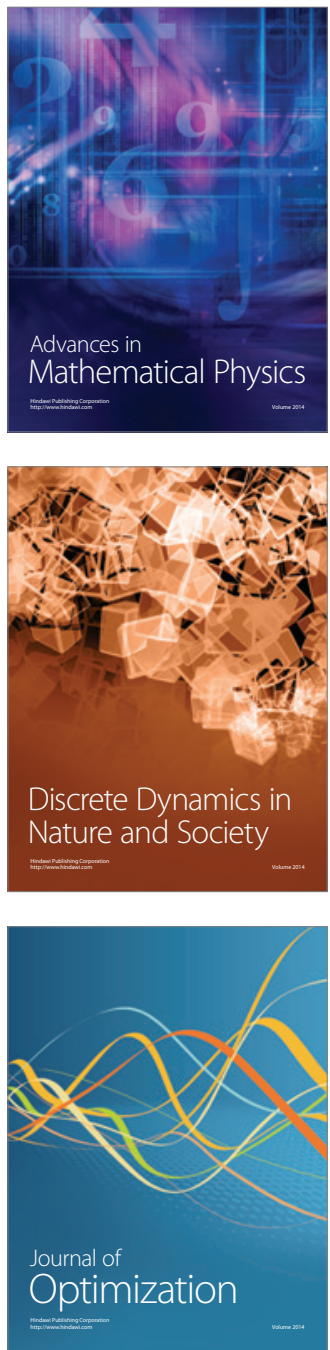